\title{
Método Linear Nodal no Esquema Iterativo NBI ${ }^{1}$
}

J.H. ZANI 2 , Fundação Educacional Serra dos Órgãos, FESO, 25964-000 Alto Teresópolis 111, RJ, Brasil.

Resumo. Este trabalho investiga a performance do esquema iterativo de inversão por blocos de um nodo, arquitetado com as equações discretizadas do método linear nodal. O método é aplicado a um problema de transporte de nêutrons, em meio nãomultiplicativo, a um grupo de energia, considerando-se espalhamento isotrópico. A modelagem matemática trata da migração dos nêutrons na formulação de ordenadas discretas, em geometria bidimensional e em sistemas de coordenadas Cartesianas. Resultados numéricos para o problema-modelo ilustram o desempenho computacional do método linear nodal no esquema iterativo de inversão por blocos.

\section{Introdução}

A equação de transporte de nêutrons independente do tempo que considera a distribuição das partículas em meio não multiplicativo, a um grupo de energia, com espalhamento isotrópico, na formulação de ordenadas discretas $\left(\mathrm{S}_{N}\right)$, em geometria bidimensional e em sistemas de coordenadas Cartesianas é um conseqüente conjunto de equações diferenciais parciais (EDP) que, ao ser integrado no domínio e transversalmente, gera um sistema composto de equações lineares e algébricas de balanço espacial e de equações diferenciais ordinárias (EDO), onde o número de incógnitas é maior que o número de equações. O desconhecimento aí está em como representar exatamente as dependências espaciais dos termos de fugas transversais.

$\mathrm{Na}$ busca das equações auxiliares que tornem o sistema determinado vários métodos foram desenvolvidos. O método constante constante nodal (CCN) [1] aproxima por uma constante as dependências espaciais dos termos de fugas transversais e as dependências espaciais dos termos de fonte de espalhamento. O método linear linear nodal (LLN) [1, 4] aproxima tais dependências espaciais linearmente. O método linear nodal $(\mathbf{L N})$ é uma variação do método $\mathbf{L L N}$ onde são adotadas as aproximações de diferenças diamante (DD) $[1,4]$ para os momentos espaciais de primeira ordem. Em todos estes métodos, onde há aproximação para os termos de espalhamento, é usual arranjar-se as equações discretizadas para o esquema de iterração na fonte, esquema (SI), cf. source iteration [3]. O método espectro nodal (SGF-CN), cf. spectro Green's function-constant nodal [2], aproxima por constantes as dependências espaciais dos termos de fugas transversais, mas não utiliza aproximações para os termos de fonte de espalhamento. Neste método, as equações

\footnotetext{
${ }^{1} \mathrm{FESO}$

2jhzani@hotmail.com
} 
discretizadas são naturalmente arranjadas para o esquema de inversão por blocos de um nodo.

O desenvolvimento dado por W. F. Walters para os métodos nodais lineares [4] e o trabalho de R. C Barros no desenvolvimento do método SGF-CN [2] constituíram os precursores históricos deste desenvolvimento. Estes trabalhos motivaram a idealizacão de um método com aproximação linear para as dependências espaciais dos fluxos angulares nos termos de fugas transversais, sem aproximação espacial para os termos de fonte de espalhamento (SGF-LN), cf. spectro Green's function linear nodal. Porém, foi necessário investigar a estrutura e o desempenho do esquema iterativo de inversão por blocos quando utilizadas as aproximações lineares para as fugas transversais. A presente pesquisa concentrou-se inicialmente na arquitetura do algoritmo e no comportamento da convergência de um método com aproximação linear para as fugas transversais e para os termos de fonte de espalhamento, porém, com as equações discretizadas arranjadas para o esquema iterativo de inversão por blocos de um nodo (LN-NBI), cf. linear nodal one-node block inversion [5], pois seria relevante para o desenvolvimento futuro do método SGF-LN.

\section{Modelagem Matemática}

Emprega-se a equação de transporte de Boltzmann para nêutrons em regime estacionário, em meio não multiplicativo, a um grupo de energia, considerando espalhamento isotrópico, em geometria bidimensional, no sistema de coordenadas Cartesianas e na formulação de ordenadas discretas $\left(S_{N}\right)$. Com estas considerações e aproximações a modelagem matemática conseqüente é formada por um sistema de EDP, que estabelecem balanços espaciais das taxas de produções e perdas de nêutrons:

$$
\mu_{m} \frac{\partial}{\partial x} \psi_{m}(x, y)+\eta_{m} \frac{\partial}{\partial y} \psi_{m}(x, y)+\sigma_{t}(x, y) \psi_{m}(x, y)=S_{e s p}(x, y)+q(x, y),
$$

onde $\left.S_{\text {esp }}(x, y)=\left[\sigma_{s o}(x, y) / 4\right)\right] \sum_{n=1}^{M} \psi_{n}(x, y) \omega_{n}$.

Na equação (2.1), $m=1: M, M=(N+2) N / 2$ é o número de ordenadas discretas, $N$ é a ordem da quadratura angular, $(x, y)$ é um ponto do domínio bidimensional, $w_{n}$ representa o peso da angular associado à direção discreta $\left(\mu_{m}, \eta_{m}\right), \sigma_{t}$ é a seção de choque macroscópica total, $\sigma_{s o}$ é a componente isotrópica da seção de choque macroscópica de espalhamento, $\psi_{m}$ é o fluxo angular de nêutrons que se deslocam na direção discreta $\left(\mu_{m}, \eta_{m}\right)$ e $q$ representa outras fontes isotrópicas.

\section{Método LLN}

Para realizar uma modelagem computacional, considera-se uma malha espacial bidimensional retangular e em um nodo homogêneo, aplica-se nas equações (2.1) os 
operadores:

$$
\begin{gathered}
L_{I J}^{x y} \equiv \frac{3^{I} 3^{J}}{h_{x} h_{y}} \int_{x_{e}}^{x_{d}} d x \int_{y_{i}}^{y_{s}} d y(\bullet) p_{I}(x) p_{J}(y), \quad(I, J)=(0,0),(0,1) e(1,0) ; \\
L_{I}^{x} \equiv \frac{3^{I}}{h_{x}} \int_{x_{e}}^{x_{d}} d x(\bullet) p_{I}(x), \quad I=0,1 ; \quad L_{J}^{y} \equiv \frac{3^{J}}{h_{y}} \int_{y_{i}}^{y_{s}} d y(\bullet) p_{J}(y), \quad J=0,1 .
\end{gathered}
$$

Os índices $i, e, s$ e $d$, referem-se, respectivamente, às faces: inferior, esquerda, superior e direita do nodo da malha espacial. $p_{I}(x)$ e $p_{J}(y)$ são os polinômios:

$$
p_{0}(x)=p_{0}(y)=1, \quad p_{1}(x)=2(x-\bar{x}) / h_{x} \quad e \quad p_{1}(y)=2(y-\bar{y}) / h_{y},
$$

onde introduziu-se: $\quad \bar{x}=\left(x^{d}+x^{e}\right) / 2 \quad$ e $\quad \bar{y}=\left(y^{i}+y^{s}\right) / 2$.

As aplicações dos operadores $L_{00}^{x y}, L_{10}^{x y}$ e $L_{01}^{x y}$ nas equações (2.1), no nodo homogêneo da malha de discretização espacial, fornecem, respectivamente, as seguintes equações de balanço espacial:

$$
\begin{gathered}
\left(\psi_{m}^{d}-\psi_{m}^{e}\right) / \varepsilon_{x, m}+\left(\psi_{m}^{s}-\psi_{m}^{i}\right) / \varepsilon_{y, m}+\bar{\psi}_{m}=(\bar{S}+q) / \sigma_{T}, \\
3\left(\psi_{m}^{d}+\psi_{m}^{e}-2 \bar{\psi}_{m}\right) / \varepsilon_{x, m}+\left(\theta_{m}^{s}-\theta_{m}^{i}\right) / \varepsilon_{y, m}+\psi_{m}^{x}=S^{x} / \sigma_{T}
\end{gathered}
$$

e

$$
3\left(\psi_{m}^{i}+\psi_{m}^{s}-2 \bar{\psi}_{m}\right) / \varepsilon_{y, m}+\left(\theta_{m}^{d}-\theta_{m}^{e}\right) / \varepsilon_{x, m}+\psi_{m}^{y}=S^{y} / \sigma_{T}
$$

Nas equações (3.1) a (3.3) foram definidos:

- os momentos espaciais médios de ordem zero do fluxo angular de nêutrons nas faces horizontais $(\mathrm{H})$ e verticais $(\mathrm{V})$ do nodo

$$
\psi_{m}^{H} \equiv L_{0}^{x} \psi_{m}\left(x, y^{H}\right), \quad H=s, i \quad e \quad \psi_{m}^{V} \equiv L_{0}^{y} \psi_{m}\left(x^{V}, y\right), \quad V=d, e ;
$$

- os momentos espaciais médios de primeira ordem do fluxo angular de nêutrons nas faces $\mathrm{H}$ e $\mathrm{V}$ do nodo

$$
\theta_{m}^{H} \equiv L_{1}^{x} \psi_{m}\left(x, y^{H}\right), \quad H=s, i \quad e \quad \theta_{m}^{V} \equiv L_{1}^{y} \psi_{m}\left(x^{V}, y\right), \quad V=d, e
$$

- os momentos espaciais médios de ordem zero do fluxo angular de nêutrons no interior do nodo

$$
\bar{\psi}_{m} \equiv L_{00}^{x y} \psi_{m}(x, y)
$$

- os momentos espaciais médios de primeira ordem nas variáveis $x$ e $y$ do fluxo angular de nêutrons no interior do nodo

$$
\psi_{m}^{x} \equiv L_{10}^{x y} \psi_{m}(x, y) \quad e \quad \psi_{m}^{y} \equiv L_{01}^{x y} \psi_{m}(x, y)
$$

- a fonte de espalhamento média de ordem zero no interior do nodo 


$$
\bar{S} \equiv L_{00}^{x y} S_{e s p}(x, y)
$$

- as fontes de espalhamento médias de primeira ordem nas variáveis $x$ e $y$ no interior do nodo

$$
S^{x} \equiv L_{10}^{x y} S_{e s p}(x, y) \quad e \quad S^{y} \equiv L_{01}^{x y} S_{e s p}(x, y)
$$

- as quantidades adimensionais

$$
\varepsilon_{x, m} \equiv h_{x} \sigma_{T} / \mu_{m} \quad \text { e } \quad \varepsilon_{y, m} \equiv h_{y} \sigma_{T} / \eta_{m}
$$

As aplicações dos operadores $L_{I}^{x}$ e $L_{J}^{y}$, com $I=J=0,1$, nas equações (2.1) fornecem o seguinte sistema de EDO:

$$
\begin{gathered}
\mu_{m} \frac{d}{d x} \hat{\psi}_{m}(x)+\left(\eta_{m} / h_{y}\right)\left[\psi_{m}\left(x, y^{s}\right)-\psi_{m}\left(x, y^{i}\right)\right]+\sigma_{T} \hat{\psi}_{m}(x)=\left(\sigma_{s o} / 4\right) \sum_{n=1}^{M} \omega_{n} \hat{\psi}_{n}(x)+q \\
\mu_{m} \frac{d}{d x} \hat{\theta}_{m}(x)+\sigma_{T} \hat{\theta}_{m}(x)= \\
6\left(\eta_{m} / h_{y}\right)\left[\hat{\psi}_{m}(x)-\frac{1}{2}\left(\psi_{m}\left(x, y^{s}\right)-\frac{1}{2}\left(\psi_{m}\left(x, y^{i}\right)\right]+\left(\sigma_{s o} / 4\right) \sum_{n=1}^{M} \omega_{n} \hat{\theta}_{n}(x)\right.\right. \\
\eta_{m} \frac{d}{d y} \tilde{\psi}_{m}(y)+\left(\mu_{m} / h_{x}\right)\left[\psi_{m}\left(x^{d}, y\right)-\psi_{m}\left(x^{e}, y\right)\right]+\sigma_{T} \tilde{\psi}_{m}(y)=\left(\sigma_{s o} / 4\right) \sum_{n=1}^{M} \omega_{n} \tilde{\psi}_{n}(y)+q
\end{gathered}
$$

e

$$
\begin{gathered}
\eta_{m} \frac{d}{d y} \tilde{\theta}_{m}(y)+\sigma_{T} \tilde{\theta}_{m}(y)= \\
6\left(\mu_{m} / h_{x}\right)\left[\tilde{\psi}_{m}(y)-\frac{1}{2}\left(\psi_{m}\left(x^{d}, y\right)-\frac{1}{2}\left(\psi_{m}\left(x^{e}, y\right)\right]+\left(\sigma_{s o} / 4\right) \sum_{n=1}^{M} \omega_{n} \tilde{\theta}_{n}(y),\right.\right.
\end{gathered}
$$

onde: $\hat{\psi}_{m}(x) \equiv L_{0}^{y} \psi_{m}(x, y), \quad \hat{\theta}_{m}(x) \equiv L_{1}^{y} \psi_{m}(x, y), \quad \tilde{\psi}_{m}(y) \equiv L_{0}^{x} \psi_{m}(x, y)$ e $\quad \tilde{\theta}_{m}(y) \equiv L_{1}^{x} \psi_{m}(x, y)$.

Se as dependências espaciais dos termos de fugas transversais nas faces dos nodos fossem conhecidas, as $3 M$ equações lineares e algébricas de balanço espacial mais as soluções das $4 M$ EDO, junto às condições de contorno nodais, formariam um sistema de equações lineares e algébricas a7 $M$ equações e $7 M$ incógnitas, que poderiam ser arranjadas para um esquema iterativo conveniente. Entretanto, as dependências espaciais dos termos de fugas transversais nas faces dos nodos não são conhecidas.

Para obter o conjunto de equações discretizadas do método LLN são introduzidas as seguintes aproximações lineares para as dependências espaciais dos fluxos angulares nas faces dos nodos e para as dependências espaciais das fontes de espalhamento no interior dos nodos da malha espacial: 


$$
\begin{aligned}
& \psi_{m}\left(x, y^{s}\right)=p_{0}(x) \psi_{m}^{s}+s\left(\mu_{m}\right) p_{1}(x) \theta_{m}^{s}, \quad \psi_{m}\left(x, y^{i}\right)=p_{0}(x) \psi_{m}^{i}+s\left(\mu_{m}\right) p_{1}(x) \theta_{m}^{i} \\
& \psi_{m}\left(x^{e}, y\right)=p_{0}(y) \psi_{m}^{e}+s\left(\eta_{m}\right) p_{1}(y) \theta_{m}^{e}, \quad \psi_{m}\left(x^{d}, y\right)=p_{0}(y) \psi_{m}^{d}+s\left(\eta_{m}\right) p_{1}(y) \theta_{m}^{d}
\end{aligned}
$$

e

$$
S_{e s p}(x, y)=p_{0}(x) p_{0}(y) \bar{S}+s\left(\mu_{m}\right) p_{1}(x) S^{x}+s\left(\eta_{m}\right) p_{1}(y) S^{y},
$$

onde $s\left(\mu_{m}\right)$ e $s\left(\eta_{m}\right)$ são os sinais das ordenadas discretas em cada sentido de varredura de transporte.

Integrando as equações (3.4) e (3.5) em $x^{e} \leq x \leq x^{d}$ e as equações (3.6) e (3.7) em $y^{i} \leq y \leq y^{s}$ e as manipulando junto às equações (3.1) a (3.3), obtêm-se para $\mu_{m}$ e $\eta_{m}$ positivos:

$$
\begin{aligned}
\psi_{m}^{d}= & \psi_{m}^{e} \exp \left(-\varepsilon_{x, m}\right)+Z_{0}\left(\varepsilon_{x, m}\right)\left(\varepsilon_{x, m} \bar{\psi}_{m}+\psi_{m}^{d}-\psi_{m}^{e}\right) \\
& +\left[2 Z_{1}\left(\varepsilon_{x, m}\right)-Z_{0}\left(\varepsilon_{x, m}\right)\right]\left[3\left(\psi_{m}^{d}+\psi_{m}^{e}-2 \bar{\psi}_{m}\right)+\varepsilon_{x, m} \psi_{m}^{x}\right], \\
\theta_{m}^{d}= & \theta_{m}^{e} \exp \left(-\varepsilon_{x, m}\right)+Z_{0}\left(\varepsilon_{x, m}\right)\left(\varepsilon_{x, m} \psi_{m}^{y}+\theta_{m}^{d}-\theta^{e}\right) \\
+ & {\left[2 Z_{1}\left(\varepsilon_{x, m}\right)-Z_{0}\left(\varepsilon_{x, m}\right)\right]\left[3\left(\varepsilon_{x, m} / \varepsilon_{y, m}\right)\left(2 \psi_{m}^{x}-\theta_{m}^{s}-\theta_{m}^{i}\right)\right], } \\
\psi_{m}^{s}= & \psi_{m}^{i} \exp \left(-\varepsilon_{y, m}\right)+Z_{0}\left(\varepsilon_{y, m}\right)\left(\varepsilon_{y, m} \bar{\psi}_{m}+\psi_{m}^{s}-\psi_{m}^{i}\right) \\
& +\left[2 Z_{1}\left(\varepsilon_{y, m}\right)-Z_{0}\left(\varepsilon_{y, m}\right)\right]\left[3\left(\psi_{m}^{i}+\psi_{m}^{s}-2 \bar{\psi}_{m}\right)+\varepsilon_{y, m} \psi_{m}^{y}\right]
\end{aligned}
$$

e

$$
\begin{aligned}
\theta_{m}^{s}= & \theta_{m}^{i} \exp \left(-\varepsilon_{y, m}\right)+Z_{0}\left(\varepsilon_{y, m}\right)\left(\varepsilon_{y, m} \psi_{m}^{x}+\theta_{m}^{s}-\theta_{m}^{i}\right) \\
& +\left[2 Z_{1}\left(\varepsilon_{y, m}\right)-Z_{0}\left(\varepsilon_{y, m}\right)\right]\left[3\left(\varepsilon_{y, m} / \varepsilon_{x, m}\right)\left(2 \psi_{m}^{y}-\theta_{m}^{d}-\theta_{m}^{e}\right)\right]
\end{aligned}
$$

Nas equações (3.8) a (3.11) foram introduzidos:

$$
Z_{0, m}\left(\varepsilon_{y, m}\right) \equiv\left[1-\exp \left(-\varepsilon_{y, m}\right)\right] / \varepsilon_{y, m} \quad \text { e } \quad Z_{1, m}\left(\varepsilon_{y, m}\right) \equiv\left[1-Z_{0, m}\left(\varepsilon_{y, m}\right)\right] / \varepsilon_{y, m} .
$$

As equações (3.1) a (3.3) e as equações (3.8) a (3.11) constituem o conjunto de equações lineares e algébricas de balanço espacial do método LLN para $\mu_{m}$ e $\eta_{m}>$ 0 .

Para que o texto não fique sobrecarregado será omitido a seguir o índice $m$ indicativo da direção angular.

\section{Método LN}

Obtêm-se as equações de balanço espacial, lineares e algébricas do método LN, introduzindo-se nas equações (3.9) e (3.11) as aproximações DD [4]: 


$$
\psi^{x}=\left(\theta^{s}+\theta^{i}\right) / 2 \quad e \quad \psi^{y}=\left(\theta^{d}+\theta^{e}\right) / 2
$$

Com estas aproximações as equações (3.9) e (3.11) fornecem as seguintes expressões para os momentos espaciais médios de primeira ordem no interior do nodo:

$$
\begin{aligned}
\psi^{x} & =\theta^{s}\left[Z_{1}\left(\varepsilon_{y}\right) / Z_{0}\left(\varepsilon_{y}\right)\right]+\theta^{i}\left[1-Z_{1}\left(\varepsilon_{y}\right) / Z_{0}\left(\varepsilon_{y}\right)\right], \\
\psi^{y} & =\theta^{d}\left[Z_{1}\left(\varepsilon_{x}\right) / Z_{0}\left(\varepsilon_{x}\right)\right]+\theta^{e}\left[1-Z_{1}\left(\varepsilon_{x}\right) / Z_{0}\left(\varepsilon_{x}\right)\right] .
\end{aligned}
$$

Explicitando $\varepsilon_{y} \psi^{x}$ na equação (3.2), $Z_{0}\left(\varepsilon_{y}\right) / Z_{1}\left(\varepsilon_{x}\right) \psi^{x}$ na equação (4.1) e somando estas equações, chega-se a uma expressão para $\psi^{x}$, que quando substituída na equação (3.8), fornece a forma aumentada [4]

$$
\bar{\psi}=\left(1-\alpha^{x}\right) \psi^{e} / 2+\left(1+\alpha^{x}\right) \psi^{d} / 2+C^{1 x} \theta^{i}+C^{2 x} S^{x} .
$$

Uma manipulação algébrica análoga com as equações (3.3), (4.2) e (3.10) permite obter:

$$
\bar{\psi}=\left(1-\alpha^{y}\right) \psi^{i} / 2+\left(1+\alpha^{y}\right) \psi^{s} / 2+C^{1 y} \theta^{e}+C^{2 y} S^{y} .
$$

Nas equações (4.3) e (4.4) foram definidos:

$$
\begin{aligned}
C^{1 x} \equiv-F^{x}\left[\varepsilon_{x} Z_{0}\left(\varepsilon_{y}\right) / \varepsilon_{y} Z_{1}\left(\varepsilon_{y}\right)\right], & & C^{1 y} \equiv-F^{y}\left[\varepsilon_{y} Z_{0}\left(\varepsilon_{x}\right) / \varepsilon_{x} Z_{1}\left(\varepsilon_{x}\right)\right], \\
C^{2 x} \equiv-F^{x}\left[h_{x} / \mu\right], & & C^{2 y} \equiv-F^{y}\left[h_{y} / \eta\right], \\
\alpha^{x} \equiv F^{x}\left[\varepsilon_{x}+\varepsilon_{x} Z_{0}\left(\varepsilon_{y}\right) / \varepsilon_{y} Z_{1}\left(\varepsilon_{y}\right)\right] & \text { e } & \alpha^{y} \equiv F^{y}\left[\varepsilon_{y}+\varepsilon_{y} Z_{0}\left(\varepsilon_{x}\right) / \varepsilon_{x} Z_{1}\left(\varepsilon_{x}\right)\right],
\end{aligned}
$$

onde $F^{x} \equiv\left[Z_{2}\left(\varepsilon_{x}\right)-Z_{1}\left(\varepsilon_{x}\right)\right] /\left\{\rho^{y} \frac{h_{x}}{\mu}\left[Z_{1}\left(\varepsilon_{x}\right)+2 Z_{3}\left(\varepsilon_{x}\right)-3 Z_{2}\left(\varepsilon_{x}\right)\right]-Z_{0}\left(\varepsilon_{x}\right)\right\}$,

$$
\begin{gathered}
F^{y} \equiv\left[Z_{2}\left(\varepsilon_{y}\right)-Z_{1}\left(\varepsilon_{y}\right)\right] /\left\{\rho^{x} \frac{h_{y}}{\eta}\left[Z_{1}\left(\varepsilon_{y}\right)+2 Z_{3}\left(\varepsilon_{y}\right)-3 Z_{2}\left(\varepsilon_{y}\right)\right]-Z_{0}\left(\varepsilon_{y}\right)\right\}, \\
\rho^{x} \equiv \mu Z_{0}\left(\varepsilon_{x}\right) / h_{x} Z_{1}\left(\varepsilon_{x}\right), \\
Z_{2}\left(\varepsilon_{y}\right) \equiv\left[1-2 Z_{1}\left(\varepsilon_{y}\right)\right] / \varepsilon_{y} \quad \text { e } \quad Z_{3}\left(\varepsilon_{y}\right) \equiv\left[1-3 Z_{0}\left(\varepsilon_{y}\right) / h_{y} Z_{1}\left(\varepsilon_{y}\right)\right] / \varepsilon_{y} .
\end{gathered}
$$

\section{Método LN no Esquema de Iteração na Fonte}

Para obter o conjunto de equações para varredura de transporte de primeiro quadrante, constituintes do esquema SI, escrevem-se as equações (4.3) e (4.4) como:

$$
\begin{aligned}
& \psi^{d}=\left[2 \bar{\psi}-\left(1-\alpha^{x}\right) \psi^{e}-2 C^{1 x} \theta^{i}-2 C^{2 x} S^{x}\right] /\left(1+\alpha^{x}\right), \\
& \psi^{s}=\left[2 \bar{\psi}-\left(1-\alpha^{y}\right) \psi^{i}-2 C^{1 y} \theta^{e}-2 C^{2 y} S^{y}\right] /\left(1+\alpha^{y}\right) .
\end{aligned}
$$


Em seguida, substituem-se as equações (5.1) e (5.2) na equação (3.1). Este procedimento fornece uma expressão que permite avaliar o fluxo angular médio

$$
\begin{aligned}
\bar{\psi}= & \left\{\left[\left(1-\alpha^{x}\right) \delta^{x}+\left(1 / \varepsilon_{x}\right)\right] \psi^{e}+\left[\left(1-\alpha^{y}\right) \delta^{y}+\left(1 / \varepsilon_{y}\right)\right] \psi^{i}+\left(2 C^{1 x} \delta^{x}\right) \theta^{i}\right. \\
+ & \left.+2 C^{1 y} \delta^{y} \theta^{e}+2 C^{2 x} \delta^{x} S^{x}+2 C^{2 y} \delta^{y} S^{y}+(\bar{S}+q) / \sigma_{T}\right\} /\left[2\left(\delta^{x}+\delta^{y}\right)+1\right] .
\end{aligned}
$$

Aqui foram definidos: $\delta^{x} \equiv 1 / \varepsilon_{x}\left(1+\alpha^{x}\right) \quad$ e $\quad \delta^{y} \equiv 1 / \varepsilon_{y}\left(1+\alpha^{y}\right)$.

Então são estimados os momentos angulares médios emergentes de ordem zero nas faces dos nodos $\psi^{d}$ e $\psi^{s}$, através das equações (5.1) e (5.2).

No próximo passo, avaliam-se os momentos:

$$
\begin{aligned}
\psi^{x} & =\left[3\left(2 \bar{\psi}-\psi^{d}-\psi^{e}\right)+\left(\theta^{i} \rho^{y}+S^{x}\right) h_{x} / \mu\right] /\left(\varepsilon_{x}+\rho^{y} h_{x} / \mu\right) ; \\
\psi^{y} & =\left[3\left(2 \bar{\psi}-\psi^{i}-\psi^{s}\right)+\left\{\theta^{e} \rho^{x}+S^{y}\right\} h_{y} / \eta\right] /\left(\varepsilon_{y}+\rho^{x} h_{y} / \eta\right) ; \\
\theta^{s} & =\psi^{x}\left[Z_{0}\left(\varepsilon_{y}\right) / Z_{1}\left(\varepsilon_{y}\right)\right]+\theta^{i}\left[1-Z_{0}\left(\varepsilon_{y}\right) / Z_{1}\left(\varepsilon_{y}\right)\right] ; \\
\theta^{d} & =\psi^{y}\left[Z_{0}\left(\varepsilon_{x}\right) / Z_{1}\left(\varepsilon_{x}\right)\right]+\theta^{e}\left[1-Z_{0}\left(\varepsilon_{x}\right) / Z_{1}\left(\varepsilon_{x}\right)\right] .
\end{aligned}
$$

As expressões para $\psi^{x}$ e $\psi^{y}$ são obtidas respectivamente através das manipulações algébricas das equações (3.2) e (4.1); (3.3) e (4.2).

Finalmente, atualizam-se os sinais dos momentos de primeira ordem:

$$
\psi^{x}=s(\mu) \psi^{x}, \quad \psi^{y}=s(\eta) \psi^{y}, \quad \theta^{s}=s(\mu) \theta^{s} \quad \text { e } \quad \theta^{d}=s(\eta) \theta^{d} .
$$

Os sinais das ordenadas discretas $s(\mu)$ e $s(\eta)$ têm o objetivo de preservar as equações de balanço espacial nos demais sentidos de varreduras de transporte.

Após percorrer os nodos da malha espacial nos quatro sentidos de varreduras de transporte, atualiza-se as fontes de espalhamento $\bar{S}, S^{x}$ e $S^{y}$.

\section{Método LN no Esquema Iterativo NBI}

Para obter o conjunto de equações discretizadas do método LN no esquema iterativo NBI parte-se das equações (3.1) a (3.3) e introduzem-se as expressões para $\psi^{x}, \psi^{y} \mathrm{e}$ $\bar{\psi}$ dadas pelas equações (4.1) a (4.4). Para quadratura $\mathrm{S}_{2}$ e.g., com auxílio da Figura 1, são arranjadas inicialmente as equações de balanço espacial para os momentos médios emergentes nas faces adjacentes aos vértices $1 \mathrm{Q}$ e $3 \mathrm{Q}$ :

Após manipular algebricamente o sistema formado pelas equações (6.1) e (6.2):

$$
\begin{array}{r}
\mathbf{A}^{(\mathbf{1})}\left[\begin{array}{c}
\psi_{1,4}^{d} \\
\psi_{1,2}^{s} \\
\theta_{1,4}^{s} \\
\theta_{1,2}^{s}
\end{array}\right]-\mathbf{B}^{(\mathbf{1})}\left[\begin{array}{c}
\psi_{2,3}^{e} \\
\psi_{4,3}^{i} \\
\theta_{2,3}^{e} \\
\theta_{4,3}^{i}
\end{array}\right]=\mathbf{C}^{(\mathbf{1})}\left[\begin{array}{c}
\psi_{1,4}^{e} \\
\psi_{1,2}^{i} \\
\theta_{1,4}^{e} \\
\theta_{1,2}^{i}
\end{array}\right]+\mathbf{D}^{(\mathbf{1})}\left[\begin{array}{c}
\psi_{2,3}^{d} \\
\psi_{4,3}^{s} \\
\theta_{2,3}^{d} \\
\theta_{4,3}^{s}
\end{array}\right]+\overrightarrow{\mathbf{F} q,} \\
\mathbf{A}^{(\mathbf{3})}\left[\begin{array}{c}
\psi_{3,2}^{e} \\
\psi_{3,4}^{i} \\
\theta_{3,2}^{e} \\
\theta_{3,4}^{i}
\end{array}\right]-\mathbf{B}^{(\mathbf{3})}\left[\begin{array}{c}
\psi_{4,1}^{d} \\
\psi_{2,1}^{s} \\
\theta_{4,1}^{d} \\
\theta_{2,1}^{s}
\end{array}\right]=\mathbf{C}^{(\mathbf{3})}\left[\begin{array}{c}
\psi_{3,2}^{d} \\
\psi_{3,4}^{s} \\
\theta_{3,2}^{d} \\
\theta_{3,4}^{s}
\end{array}\right]+\mathbf{D}^{(\mathbf{3})}\left[\begin{array}{c}
\psi_{4,1}^{e} \\
\psi_{2,1}^{i} \\
\theta_{4,1}^{e} \\
\theta_{2,1}^{i}
\end{array}\right]+\overrightarrow{\mathbf{F}} q,
\end{array}
$$




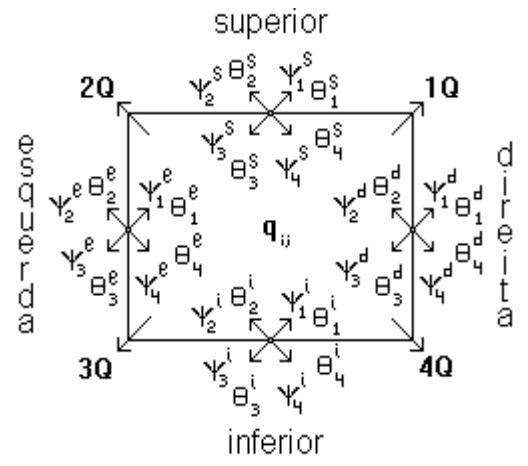

Figura 1: Momentos espaciais incidentes e emergentes nas faces do nodo.

são obtidas as entradas das matrizes resposta $\mathbf{G}^{+}, \mathbf{G}^{-}$e $\mathbf{S}$, com todos os momentos espaciais do fluxo angular médio nas faces, emergentes dos nodos, explícitos em função de todos os momentos espaciais médios incidentes nas faces do nodo e das fontes interiores do nodo da malha espacial.

$$
\left[\begin{array}{c}
\psi_{1,4}^{d} \\
\psi_{1,2}^{s} \\
\theta_{1,4}^{d} \\
\theta_{1,2}^{s}
\end{array}\right]=\mathbf{G}^{(\mathbf{1}+)}\left[\begin{array}{c}
\psi_{1,4}^{e} \\
\psi_{1,2}^{i} \\
\theta_{1,4}^{e} \\
\theta_{1,2}^{i}
\end{array}\right]+\mathbf{G}^{(\mathbf{1}-)}\left[\begin{array}{c}
\psi_{2,3}^{d} \\
\psi_{4,3}^{s} \\
\theta_{2,3}^{d} \\
\theta_{4,3}^{s}
\end{array}\right]+\mathbf{S}^{(\mathbf{1})} \overrightarrow{\mathbf{F}} q
$$

onde $\mathbf{G}^{(\mathbf{1}+)}=\left\{\mathbf{A}^{(\mathbf{1})}-\mathbf{B}^{(\mathbf{1})} \mathbf{E}\left[\mathbf{A}^{(\mathbf{3})}\right]^{-} \mathbf{B}^{(\mathbf{3})} \mathbf{E}\right\}^{-}\left\{\mathbf{C}^{(\mathbf{1})}+\mathbf{B}^{(\mathbf{1})} \mathbf{E}\left[\mathbf{A}^{(\mathbf{3})}\right]^{-} \mathbf{D}^{(\mathbf{3})} \mathbf{E}\right\}$, $\mathbf{G}^{(\mathbf{1}-)}=\left\{\mathbf{A}^{(\mathbf{1})}-\mathbf{B}^{(\mathbf{1})} \mathbf{E}\left[\mathbf{A}^{(\mathbf{3})}\right]^{-} \mathbf{B}^{(\mathbf{3})} \mathbf{E}\right\}^{-}\left\{\mathbf{D}^{(\mathbf{1})}+\mathbf{B}^{(\mathbf{1})} \mathbf{E}\left[\mathbf{A}^{(3)}\right]^{-} \mathbf{C}^{(3)} \mathbf{E}\right\}$, e $\quad \mathbf{S}^{(1)}=\left\{\mathbf{A}^{(1)}-\mathbf{B}^{(1)} \mathbf{E}\left[\mathbf{A}^{(3)}\right]^{-} \mathbf{B}^{(3)} \mathbf{E}\right\}^{-}\left\{\mathbf{I}^{(1)}+\mathbf{B}^{(1)} \mathbf{E}\left[\mathbf{A}^{(3)}\right]^{-}\right\}$.

$$
\left[\begin{array}{c}
\psi_{3,2}^{e} \\
\psi_{3,4}^{i} \\
\theta_{3,2}^{e} \\
\theta_{3,4}^{i}
\end{array}\right]=\mathbf{G}^{(\mathbf{3}+)}\left[\begin{array}{c}
\psi_{3,2}^{d} \\
\psi_{3,4}^{s} \\
\theta_{3,2}^{d} \\
\theta_{3,4}^{s}
\end{array}\right]+\mathbf{G}^{(\mathbf{3}-)}\left[\begin{array}{c}
\psi_{4,1}^{e} \\
\psi_{2,1}^{i} \\
\theta_{4,1}^{e} \\
\theta_{2,1}^{i}
\end{array}\right]+\mathbf{S}^{(\mathbf{3})} \overrightarrow{\mathbf{F}} q
$$

$$
\begin{aligned}
& \text { onde } \mathbf{G}^{(3+)}=\left\{\mathbf{A}^{(\mathbf{3})}-\mathbf{B}^{(\mathbf{3})} \mathbf{E}\left[\mathbf{A}^{(\mathbf{1})}\right]^{-} \mathbf{B}^{(\mathbf{1})} \mathbf{E}\right\}^{-}\left\{\mathbf{C}^{(\mathbf{3})}+\mathbf{B}^{(\mathbf{3})} \mathbf{E}\left[\mathbf{A}^{(\mathbf{1})}\right]^{-} \mathbf{D}^{(\mathbf{1})} \mathbf{E}\right\} \\
& \text { e } \quad \mathbf{G}^{(\mathbf{3}-)}=\left\{\left(\mathbf{A}^{(\mathbf{3})}-\mathbf{B}^{(\mathbf{3})} \mathbf{E}\left[\mathbf{A}^{(\mathbf{1})}\right]^{-} \mathbf{B}^{(\mathbf{1})} \mathbf{E}\right\}^{-}\left\{\mathbf{D}^{(\mathbf{3})}+\mathbf{B}^{(\mathbf{3})} \mathbf{E}\left[\mathbf{A}^{(\mathbf{1})}\right]^{-} \mathbf{C}^{(\mathbf{1})} \mathbf{E}\right\}\right. \\
& \mathbf{S}^{(\mathbf{3})}=\left\{\mathbf{A}^{(\mathbf{3})}-\mathbf{B}^{(\mathbf{3})} \mathbf{E}\left[\mathbf{A}^{(\mathbf{1})}\right]^{-} \mathbf{B}^{(\mathbf{1})} \mathbf{E}\right\}^{-}\left\{\mathbf{I}^{(\mathbf{1})}+\mathbf{B}^{(\mathbf{3})} \mathbf{E}\left[\mathbf{A}^{(\mathbf{1})}\right]^{-}\right\}
\end{aligned}
$$

As entradas das matrizes $\mathbf{G}^{+}, \mathbf{G}^{-}$e $\mathbf{S}$, para o cálculo dos momentos médios emergentes nas faces adjacentes aos vértices $2 \mathrm{Q}$ e $4 \mathrm{Q}$ são construídas de forma análoga. Os sinais negativos sobrescritos após as operações matriciais indicam a operação matricial inversa, exceto nas matrizes $\mathbf{G}^{+} \mathrm{e} \mathbf{G}^{-}$onde caracterizam as contribuições dos momentos espaciais incidentes nas faces. De uma forma geral, para quadratura $S_{N}$, as matrizes $\mathbf{A}^{(k)}, \mathbf{B}^{(k)}, \mathbf{C}^{(k)}, \mathbf{D}^{(k)}, \mathbf{G}^{(k+)}, \mathbf{G}^{(k-)}$ e $\mathbf{S}^{(k)}$ são de ordem $2 M \times 2 M$. k representa os sentidos de varreduras de transporte, $\mathrm{k}=1: 4$, e $M$ é o número de sentidos de discretização angular, $M=N(N+2) / 2$. Os vetorescoluna são de ordem $2 M ; M$ momentos espaciais de ordem zero do fluxo angular de nêutros e $M$ momentos de primeira ordem. Para quadratura $S_{4}$, e.g., cada elemento de matriz, tomando-se a quadratura $S_{2}$ como referência, estende-se para um 
bloco de matriz $3 \times 3$. Estes blocos têm dimensões dadas pelo número de ordenadas discretas por quadrante, i.e., $M=N(N+2) / 8$. A matriz $\mathbf{E}$ é uma matriz elementar e foi introduzida apenas para operar mudanças convenientes na ordem das componentes dos vetores-coluna. $\overrightarrow{\mathbf{F}}$ é um vetor coluna com $2 M$ linhas, com as componentes $\mathrm{f}_{p, 1}$ tais que: $\mathrm{f}_{p, 1}=1$ se $\mathrm{p}$ menor ou igual a $M$ e $\mathrm{f}_{p, 1}=0$ para o restante.

As matrizes $\mathbf{G}^{+}, \mathbf{G}^{-}$e $\mathbf{S}$ carregam informações sobre os parâmetros materiais, as ordenadas discretas, a largura e a altura dos nodos. São calculadas apenas uma vez para um nodo de cada zona material homogênea, antes de se iniciar o processo iterativo.

\section{Resultados Numéricos}

Para ilustrar o mérito do esquema iterativo de inversão nodal, foram gerados resultados numéricos para um problema-modelo de fonte fixa. A Figura 2 representa um poço de sondagem geofísica para identificação de recursos geológicos, cf. Nuclear well-logging [1]. No poço é inserido um cilindro de aço contendo dois detectores: $\mathrm{D}_{1}$ e $\mathrm{D}_{2}$. As condições de contorno utilizadas foram as de vácuo e reflexiva do transporte.
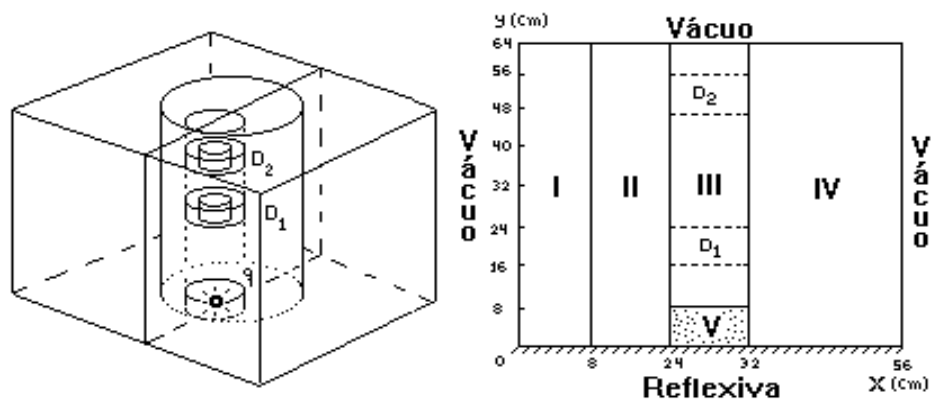

Figura 2: Poço de sondagem geofísica.

Os parâmetros das zonas materiais I, II, III e IV estão listados na Tabela 1. A fonte isotrópica e unitária de nêutrons está uniformemente distribuída na região V.

\begin{tabular}{cccc}
\hline \hline $\begin{array}{c}\text { Parâmetros } \\
\left(\mathrm{cm}^{-1}\right)\end{array}$ & I e IV & $\begin{array}{c}\text { Zonas Materiais } \\
\text { II }\end{array}$ & III e V \\
\hline$\sigma_{S}$ & 0,314419 & 0,634883 & 0,494460 \\
\hline$\sigma_{T}$ & 0,330263 & 0,694676 & 0,499122 \\
\hline \hline
\end{tabular}

Tabela 1: Parâmetros das zonas materiais.

Os resultados numéricos obtidos para este problema-modelo foram gerados em um computador com 256 Mbytes-RAM, 32-bit e $1180 \mathrm{MHz}$, utilizando-se a quadratura angular $S_{6}$ de simetria de nível [3] e o critério de convergência de $10^{-5}$ na norma máxima do vetor desvio relativo percentual do fluxo escalar médio, nos nodos da malha espacial, avaliados a cada duas estimativas consecutivas. 
A Tabela 2 lista as malhas de discretizações com partições uniformes nas orientações espaciais (ME), os fluxos escalares médios $(\Phi)$ nas regiões dos detectores $\mathrm{D}_{1}$ e $\mathrm{D}_{2}$, os desvios relativos percentuais (\%) em relação ao método de malha fina DD-SI e o número de iterações (NI).

\begin{tabular}{ccccccc}
\hline \hline & ME & \multicolumn{3}{c}{$\Phi\left(\mathrm{cm}^{-2} \cdot \mathrm{s}^{-1}\right)$} & & \\
\hline & & $\mathrm{D}_{1}$ & $\%$ & $\mathrm{D}_{2} \times 10^{-2}$ & $\%$ & \\
\hline LN-SI: & $7 \times 8$ & 1,647 & $-3,97$ & 1,374 & 8,19 & 315 \\
\hline & $14 \times 16$ & 1,732 & 0,99 & 1,260 & $-0,79$ & 327 \\
\hline & $28 \times 32$ & 1,717 & 0,12 & 1,247 & $-1,81$ & 329 \\
\hline LN-NBI: & $7 \times 8$ & 1,590 & $-7,29$ & 1,062 & $-16,34$ & 33 \\
\hline & $14 \times 16$ & 1,698 & $-0,99$ & 1,230 & $-3,15$ & 47 \\
\hline & $28 \times 32$ & 1,705 & $-0,58$ & 1,238 & $-2,52$ & 66 \\
\hline DD-SI: & $140 \times 160$ & 1,715 & - & 1,270 & - & 278 \\
\hline \hline
\end{tabular}

Tabela 2: Performance dos métodos: LN-SI e LN-NBI em relação ao DD-SI.

\section{Discussões}

Devido ao grande esforço algébrico para obterem-se as equações discretizadas do método LN para o sentido de varredura de primeiro quadrante, as equações para os demais sentidos de varredura podem ser obtidas através de argumentos de simetria espacial, i.e., preservando-se os pesos dos momentos espaciais de ordem zero que são incidentes e emergentes nas faces do nodo, em relação aos sentidos de varredura de transporte e atendendo para os sinais dos momentos espaciais e fontes de primeira ordem.

Os resultados numéricos listados na Tabela 2, mostram que os desvios relativos percentuais obtidos para os métodos LN-SI e LN-NBI ao serem comparados aos gerados com o método e esquema iterativo DD-SI, em geral, aumentam com malhas espaciais maiores. Nestes métodos, as aproximações adotadas para as dependências espaciais das fugas transversais e das fontes de espalhamento são as responsáveis por estes resultados numéricos não serem livres de erro de truncamento espacial. Observa-se na Tabela 2 que, o número de iterações para o algoritmo do esquema iterativo SI apresenta-se pouco sensível ao alargamento da malha espacial, enquanto o número de iterações do esquema iterativo NBI diminui com o alargamento da malha espacial, o que aponta para utilização de malhas espaciais relativamente mais grossas em métodos livres de erro de truncamento espacial, arranjados para o esquema iterativo NBI.

O arranjo matemático do sistema de equações lineares e algébricas do método LN para o esquema iterativo NBI foi o primeiro passo para o desenvolvimento futuro do método SGF-LN, que espera-se ser mais preciso que o método $\mathbf{L N}$ por não utilizar aproximações para as fontes de espalhamento e, conseqüentemente, permita a utilização de malhas espaciais relativamente mais grossas. 


\section{Agradecimentos}

Marcos Pimenta de Abreu (D.Sc.), Ricardo Carvalho de Barros (Ph.D.), IPRJ/UERJ, Rio de Janeiro, Brasil e Todd Wareing (D.Sc.), Los Alamos National Laboratory, EUA.

Abstract. This paper describe de architecture and examines the performance of the one-node block inversion (NBI) iterative schemes, solve the discretized equations of the linear nodal method $(\mathbf{L N})$ in one-speed neutron transport, with isotropic scattering and that dislocates in non multiplicative region. The mathematical model treat the transport equations in X, Y geometry, Cartesian coordinates systems and in the discrete ordinates $\left(S_{N}\right)$ formulation. The numerical results illustrate the computational performance of the iterative schemes for typical stead-state model problem.

\section{Referências}

[1] Y.Y. Azmy, Comparison of three approximations to the linear-linear nodal transport method in weighted diamond-difference form, Nuclear Science and Engineering, 100 (1988), 190-200.

[2] R.C. Barros, "A Spectral Nodal Method for the Solution of Discrete Ordinates Problems in One-and Two-Dimensional Cartesian Geometry", Ph.D. dissertation, The University of Michigan, Ann Arbor, Michigan, 1990.

[3] E.E. Lewis e W.F.Jr. Miller, "Computational Methods of Neutron Transport", American Nuclear Society, La Grange Park, Illinois, 1993.

[4] W.F. Walters, Augmented weighted diamond form of the linear nodal scheme for Cartesian coordinates systems, American Nuclear Society, 2 (1985), 452460 .

[5] J.H. Zani, "Esquemas Iterativos para Solução Numérica Nodal de Problemas de Transporte de Nêutrons Monoenergéticos na Formulação $S_{N}$ em Geometria Bidimencional Cartesiana", Tese de doutorado, UERJ, IPRJ, Nova Friburgo, Rio de Janeiro, 2001. 
